\title{
First Isolation of Enterovirus 71 (EV-71) From Northern Brazil
}

Leticia Martins Lamarão, Ana Maria T. R. Maciel and Maria de Lourdes Contente Gomes
Evandro Chagas Institute, Epidemiology Centre of State of Pará, National Foundation of Health, Belém, PA, Brazil

\begin{abstract}
Enterovirus 71 (EV-71) has been associated to cases of neurological disease in many countries including Brazil. This virus has now been reported from three of the five Brazilian regions. Our study relates the findings concerning to the first isolate of this virus in Northern region of Brazil. A 15-month old female patient, from the rural zone of the municipality of Santana do Araguaia in southern Pará state was admitted at the hospital with acute, flaccid, asymmetric and ascending motor deficiency, located in the right lower limb. Stools samples from this child were inoculated in RD cells and was isolated an EV-71. We plan to sequence our strain and compare it to other isolates in Brazil. Differences at the molecular level can explain why EV-71 strains circulating in other continents, such as Asia, appear to be more virulent.

Key Words: Enterovirus-71, neurological disease.
\end{abstract}

Enterovirus 71 (EV-71) was identified as a new type of enterovirus by Schmidt et al. [1] in 1974. They reported the isolation of $20 \mathrm{EV}-71$ strains from patients with acute central nervous system (CNS)disease. Other studies reported an association of EV-71 with cases of paralytic disease, similar to poliomyelitis, occurring in New York [2], as well as an epidemic of acute disease of the CNS in Hungary [3]. An association of EV-71 with neurological disease, throughout a 14year period in the United States, was reported by Alexander et al. [4]. This virus was identified in 193 isolates between 1985 and 1989. The patients' ages ranged from neonates to 46 years; most of them were less than five years old. Outbreaks of hand, foot and mouth disease caused by EV-71 were reported by Lum et al. [5] inMalaysia in 1998 and by Monto et al. [6] in Taiwan in1999. In the Malaysian study, EV-71 strains were isolated from four children who died. Post-

Received on 10 April 2003; revised 16 June 2003.

Address for correspondence: Dr. Maria de Lourdes C. Gomes, Instituto Evandro Chagas, Av. Almirante Barroso 492, 66090000 Belém, Pará, Brasil. Phone: 91 2142018. Fax 91 2142005. email: lourdesgomes@iec.pa.gov.br

The Brazilian Journal of Infectious Diseases 2003;7(4):278-281 (C) 2003 by The Brazilian Journal of Infectious Diseases and Contexto Publishing. All rights reserved. mortem studies showed infection by EV-71, with extensive damages to the medulla and the pons. In the Taiwan study, 405 patients were examined, 78 of whom died; $92 \%$ of these were infected by EV-71.

The first report of EV-71 in Brazil was made in 1990 by da Silva et al. [7], in 1990. They studied sera from 28 patients with symptoms of either AFP (sudden paralysis, possible polio) or paresy, including three cases of patients with facial palsy; none of them had been infected by poliovirus. Neutralizing antibodies for EV-71 were found in nine patients (32\% of the cases), and three developed sequelae following infection. Takimoto et al. [8] identified EV-71 in children up to 15 years old from 1988 to 1990. Among 426 children who presented with acute neurological disease, EV-71 infection was diagnosed in $24(5.6 \%)$. Infection was confirmed by viral isolation in 13 children, both viral isolation and serological tests in four children, and serology alone in seven children. This virus was also isolated from samples of $15(3.5 \%)$ contacts of 165 patients. Seven of the 24 children infected by EV-71 had sequelae, consisting of motor deficiency, when they were examined six months following the onset of disease. We report the clinical and epidemiological findings of the first isolation of EV-71 in northern Brazil. 
Figure 1. Enterovirus-specific fluorescence in an RD (human embryonal) cell layer
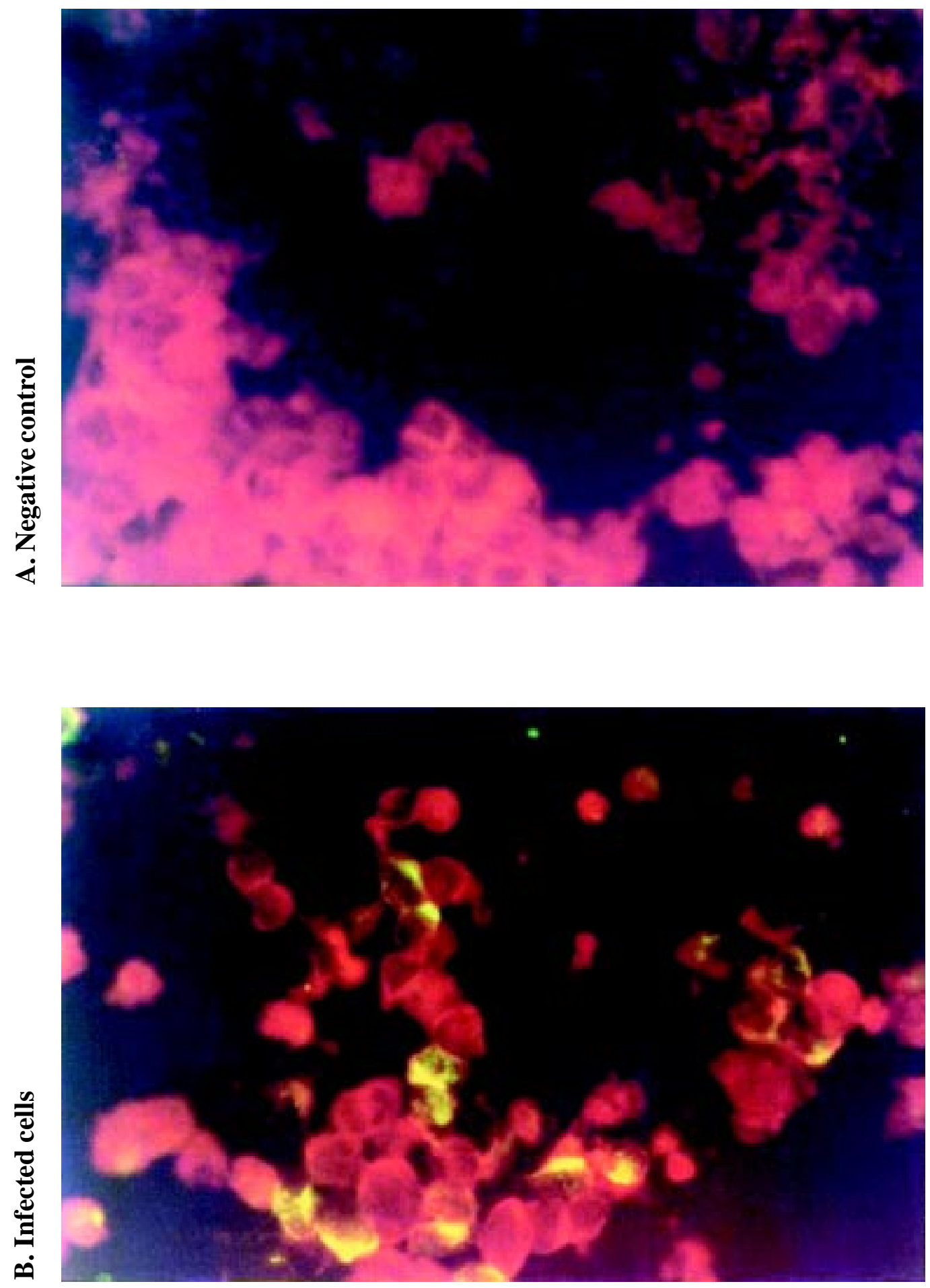
A 15-month old female patient, from the rural zone of the municipality of Santana do Araguaia, located in southern Pará state, in northern Brazil, was admitted at the "Hospital Municipal São Francisco de Assis", Santana do Araguaia, on December 16, 1999, with symptoms of fever and paresy of abdominal muscles that began six days before; acute, flaccid, asymmetric and ascending motor deficiency, located in the right lower limb (RLL) that had begun four days before was also identified. At physical examination hypotonia of the RLL was noted. Sensorial reflexes were normal and meningeal irritation signs were absent. The patient had received three doses of Sabin vaccine, dated November 6, 1998, January 13, 1999 and March 30, 1999. The following diagnosis hypotheses were considered: Guillain Barré Syndrome, poliomyelitis and congenital luxation. According to the parents, she had not traveled during the 30 days before the onset of motor deficiency and had no close contacts with persons coming from other localities. Possible toxic mechanisms for the motor deficiency, such as mercury contamination or drug toxicity, were ruled out. No history of intramuscular injection or traumatism was reported. Congenital luxation was excluded following $\mathrm{x}$-ray examination of the pelvis.

The virus was isolated from stool samples, prepared with balanced salt solution (Hank's BSS) plus antibiotics (streptomycin, gentamicin and penicillin) and fungicide (B Amphotericin) and inoculated into RD (human embryonal) cells, maintained in Eagle's minimum essential medium (MEM). The CPE (cytopathic effect) was observed after two passages, following a seven-day incubation period in each. Fluorescence specific for EV-71 was found in stool samples (Figure 1). No neutralization was obtained with poliovirus antisera (Centers for Disease Control and Prevention, Atlanta, GA, USA).

Many studies have stressed the association of EV71 with cases of AFP, mainly in other countries. Hanson et al. [9] mentioned that an 18-month-old child presenting with severe weakness in his left leg was admitted to a medical center in central Mississippi. Neurologic examination was normal, except that left hip and thigh muscles were not functioning. The left knee-jerk was absent. A follow-up examination months later showed a partial recovery of function in the lower left leg. Stool specimens yielded EV-71. Our patient was admitted to a hospital presenting with acute motor deficiency in the RLL. Her right knee-jerk was decreased. Six months later her muscle power and tonus were normal, different from what was reported by Hanson et al. [9]. Another case, reported by Chonmaitree et al. [2] involved a two-year-old girl who was admitted to the hospital because of fever with neck stiffness, and an inability to bear weight on her right leg during one day. Muscle power of both hips and thighs was decreased asymmetrically, the right being weaker than the left. She could not bear weight on her right leg. Deep tendon reflexes of both lower extremities were diminished; neither knee nor ankle jerk was detectable on the right side. Attempts at viral isolation from nasopharynx/throat samples and stools yielded EV-71. Four months after the acute illness her right leg was normal, with no residual weakness. One year later this patient had no apparent sequelae. This finding is similar to ours, because six months after the onset of the symptoms our patient also had no sequelae. The viral strain that we isolated could be similar to that isolated by Chonmaitre et al. [2], considering that, in both cases, the children had no sequelae at physical examination made months later. On the other hand, the strains infecting the children mentioned in the studies of Takimoto et al. [8] and Hanson et al. [9] may be different, because those children had motor deficiencies at physical exam made several months later.

Since several aspects of EV-71 infection in Brazil are unknown, further studies are very important. This is the first report of the occurrence of EV-71 in northern Brazil. This virus has now been reported from three of the five Brazilian regions. We plan to sequence our strain and compare it to other isolates in Brazil. We seek to determine whether differences at a molecular level can explain why EV-71 strains circulating in other continents, such as Asia, appear to be more virulent.

\section{Acknowledgements}

We thank Dr. Alexandre C. Linhares for critically reviewing this manuscript. 


\section{References}

1. Schmidt NJ., Lennette EH., Ho HH. An apparently new enterovirus isolated from patients with disease of central nervous system. Journal of Infectious Diseases 1974;129: 304-9.

2. Chonmaitree T., Menegus MA., Schervish-Swierkosz EM., Schwalenstocker BA. Enterovirus 71 infection: report of an outbreak with two cases of paralysis and a review of the literature. Pedriatrics 1981; 67: 489-493.

3. Nagy G., Takátsy S., Kurán E. et al. Virological diagnosis of enterovirus type 71 infections: experiences gained during an epidemic of acute CNS diseases in Hungary in 1978. Archives of Virology 1982;71:217-27.

4. Alexander JP., Baden L., Pallansch MA., Anderson LJ. Enterovirus 71 infections and neurologic disease- United States 1985-1989. The Journal of Infectious Diseases 1994; 169:905-908.

5. Lum LCS., Wong KT., Lam SK. et al. Fatal enterovirus 71 encephalomyelitis. The Journal of Pediatrics 1998;133(6): 795-798.

6. Monto H., Eng-Rin C., Kwo-Hsiung H. et al. An epidemic of enterovirus 71 infection in Taiwan. The New England Journal of Medicine 1999;341: (N13) 929-935.

7. Da Silva EE., Fillipis AM., Schatzmayr HG., Cortes LC. Evidence of enterovirus 71 infection in Brazil. Memórias do Instituto Oswaldo Cruz 1990;85: 131-40.

8. Takimoto S., Waldman EA., Moreira RC. et al. Enterovirus 71 infection and acute neurological disease among children in Brazil (1988-1990). Transaction of the Royal Society of Tropical Medicine and Hygiene 1998;92: 25-28.

9. Hanson RR., Myers H., Thompson FE. Case of paralytic illness associated with Enterovirus 71 infection. MMWR 1988;37(7):107. 